\title{
One code
}

Genomic research, sensitively deployed, has enormous potential to improve human health, animal health and agricultural crop quality and to guide sustainable contributions to the health of our environment. Within this broad context, we can learn from the metabolic adaptations and vulnerabilities of species threatened by environmental challenges in the context of climate change.

ife can be sweet for a koala, and even savored, as it carries a full complement of TAS1R genes encoding sweet and umami taste receptors-according to the first reference sequence for this unique living representative of its marsupial family by Rebecca Johnson and colleagues. Eating to live can also be all kinds of bitter, and the animal has evolved a specific set of duplicated TAS2R genes to selectively minimize its exposure to the bitter, toxic secondary metabolites from eucalyptus leaves, its staple diet. Apparently, koalas only drink water in exceptionally hot, dry weather, relying instead upon duplicated aquaporin 5 genes to gauge the water content of tasty leaves. After just a few hours a day of active search and feeding on just those leaves selected to fit its olfactory and vomeronasal (VIR) portfolio, the koala rests and sleeps while its repertory of expanded and diversified cytochrome P450 (CYP2C) liver enzymes speed through a pharmaceutical feedstock of terpenes and phenylpropanoids churned out by the similarly expanded and diversified synthase enzymes of Eucalyptus (Nature 510, 356-362, 2014).

These adaptations led to a successful expansion of the species after its first appearance in the fossil record, according to a simulation of the demographic history of the species derived from coalescent analysis of the maternal and paternal variants present in each of the three diploid koala genomes published here. After humans arrived in Australia and megafauna, including other large marsupials, became extinct, koala populations declined to their current estimated level of 144,000-605,000 individuals. Genomic variation in koalas shows that their populations in eastern Australia are structured by the natural barriers of large river valleys and that the recent translocations of the species into southeastern regions have left a large proportion of these populations lacking genetic diversity relative to the declining northeastern populations. This population structure needs to be taken into account if the species is to be conserved and is of particular importance in understanding the challenges to the koala from infectious diseases such as retroviruses and Chlamydia.

Our cover artwork, "You've got to crawl before you can climb" by Adam Douglas (http://www.blakdouglas.com.au/), features a koala, somehow reminiscent of Australia itself, resolutely making its way through leafless terrain under a relentless sun. Yes, this species declined in the Anthropocene, but it has not gone extinct, either while coexisting with Aboriginal people or being hunted for its pelt by nineteenthcentury Australians. However, without resourced corridors within which to move as conditions change, the koala becomes emblematic of the fate of indigenous people, which eventually becomes the fate of all people. Recognizing this, we advocate for judicious collection and dissemination of genomic information as a way to assess our unknown vulnerabilities and our capacity for adaptation and resilience, not only with respect to health challenges to human populations but also to the animals and plants that are co-evolving with us. This means we need more genomic data from the shared code of humans, animals and plants in the context of solving real challenges of unequal access to resources, of unfair disease burdens, in depleted as well as rich environments. The unifying principle of one genetic code is complementary to the ecological perspective of one shared system. Genomics will not by itself solve problems of resource distribution and intervention to promote human, animal and environmental health, but it underpins the set of possible solutions by pointing out the ways that biological variation influences population dynamics, metabolic adaptations and pathogenic challenges. Let us hope that our shared code can be an incentive and more widely understood as the source of adaptation of all living things to change.

Published online: 27 July 2018 https://doi.org/10.1038/s41588-018-0194-9 